\title{
Estudo do comportamento reológico de barbotinas preparadas com argilas da Formação Corumbataí utilizadas no pólo cerâmico de Santa Gertrudes (SP)
}

\author{
(Study of rheological behavior of slips prepared with clays from \\ Corumbatai Formation used in ceramic pole of Santa Gertrudes (SP))
}

\author{
R. R. Rocha, A. Zanardo, M. M. T. Moreno \\ Departamento de Petrologia e Metalogenia, IGCE - UNESP \\ Av. 24 A, 1515, Bela Vista, Rio Claro, SP, 13506-900 \\ rogers.rocha@rochaforte.com.br,azanardo@rc.unesp.br,mmoreno@rc.unesp.br
}

\begin{abstract}
Resumo
Este estudo surgiu da necessidade das indústrias cerâmicas do pólo de Santa Gertrudes (SP) se adaptarem às mudanças do comportamento do mercado e às exigências dos órgãos ambientais em relação aos riscos causados pela geração de poeira na fase de preparação da massa. Assim, o processo de preparação de massa via úmida pode ser uma alternativa para esta substituição, sendo necessária a obtenção de uma barbotina que apresente um comportamento reológico adequado ao processo e ao consumo de energia. Foram feitas medidas de viscosidade, tensão de cisalhamento e taxa de cisalhamento com viscosímetro rotacional, tendo como parâmetros de controle, o teor de sólidos da barbotina e a granulometria. Desta forma, foram verificadas variações das suspensões, como o comportamento pseudoplástico e a tixotropia. Também foi analisada a dependência do comportamento reológico com a mineralogia das argilas, obtida por difração de raios X. Os resultados das curvas reológicas mostraram-se muito coerentes com a mineralogia determinada, indicando uma piora do comportamento reológico no sentido da base para o topo da Formação Corumbataí, devido à alteração supérgena e presença de esmectitas.
\end{abstract}

Palavras-chave: matéria-prima cerâmica, Formação Corumbataí, reologia, revestimento cerâmico, moagem via úmida.

\begin{abstract}
The study of rheological behavior of clay slips at Corumbatai Formation arose from the need for the ceramic industries of Santa Gertrudes pole (SP) to adapt to the changes of the market behavior and to the demands of the environmental organs regarding the risks caused by the generation of dust in the mass preparation process. The wet grinding process can be an alternative for this substitution, and it's important to have proper rheological features of the slips for the process and energy consumption. Viscosity measurements, shear stress and shear rate have been made with rotational viscometer, by having the solid content of the slip and particle size as control parameters. Therefore variations of the suspensions have been verified, like the pseudoplastic behavior and the tixotropy. The dependence of the rheological behavior on the clay mineralogy has also been analyzed and it was obtained by X-ray diffraction. The results of the rheological curves appeared to be very coherent with the determined mineralogy, indicating a worsening of the rheological behavior from the bottom to the top of the Corumbatai Formation due to the supergenic alteration and the presence of smectites.

Keywords: ceramic raw material, Corumbataí Formation, rheology, ceramic tile, wet grinding process.
\end{abstract}

\section{INTRODUÇÃO}

Para a preparação de massa via úmida, é muito importante obter uma barbotina ou suspensão argilosa, com propriedades de fluidez e viscosidade controladas. Assim, torna-se fundamental um estudo do comportamento reológico dessas suspensões.

O modelamento que correlaciona a taxa de deformação de um fluido com a tensão externa aplicada foi desenvolvido inicialmente por Isaac Newton, que mostrou que o comportamento de fluxo de um fluido ideal (posteriormente denominado newtoniano) é descrito conforme a equação (A):

$$
\tau=\frac{\mathrm{F}_{\mathrm{t}}}{\mathrm{A}}=\eta \frac{\mathrm{dv}}{\mathrm{dx}}=\eta \gamma
$$

onde $F_{t}$ é a força tangencial aplicada sobre uma área $A$, cuja razão é conhecida como tensão de cisalhamento $(\tau),(\mathrm{dv} /$ $\mathrm{dx}$ ) o gradiente de velocidade que descreve o cisalhamento sentido pelo fluido, conhecido como taxa de cisalhamento $\left(\gamma^{\prime}\right)$. O coeficiente de proporcionalidade $\eta$ entre a taxa de cisalhamento e a tensão de cisalhamento foi denominado viscosidade [1]. O termo viscosidade pode ser entendido como, "o atrito interno de um fluido, causado pela atração molecular, o qual provoca uma resistência ao escoamento" 
[2]. Os fluidos não-newtonianos não apresentam uma relação linear entre a tensão e a taxa de cisalhamento. Neste caso, a viscosidade deixa de ser uma característica intrínseca do fluido e passa a depender da taxa de cisalhamento e de outros fatores: natureza físico-química das substâncias, temperatura, pressão, tempo e campo elétrico [3]. Assim, outras expressões matemáticas são utilizadas para descrever a viscosidade de fluidos não-newtonianos, a mais utilizada é a razão simples ( $\tau$ $\left./ \gamma^{\prime}\right)$, denominada de viscosidade aparente $\left(\eta_{\mathrm{ap}}\right)$.

As barbotinas cerâmicas podem apresentar um comportamento independente do tempo, mas dependente da taxa de cisalhamento, chamado pseudoplástico e simultaneamente um comportamento dependente do tempo conhecido como tixotropia. O comportamento pseudoplástico é verificado quando a viscosidade aparente do fluido diminui com o aumento da taxa e/ou da tensão de cisalhamento [1,2]. A equação de estado reológico mais utilizada para descrever o comportamento pseudoplástico das suspensões é dada pela seguinte lei das potências da equação (B):

$$
\tau=\mathrm{k}\left(\gamma^{\prime}\right)^{\mathrm{n}}
$$

onde $\mathrm{k}$ é uma constante (consistency index ou índice de consistência) e n (flow index) é um índice de comportamento do fluido que, para o caso de suspensões pseudoplásticas, é menor que $1[1,2]$.

A tixotropia também pode ser definida como uma transformação sol-gel isotérmica e reversível, de uma suspensão contendo argila, a suspensão torna-se menos viscosa pela agitação e mais espessa (gelifica) pelo repouso [4]. Os comportamentos dependentes do tempo são fortemente influenciados pela "história de cisalhamento" da suspensão, que consiste na seqüência e duração das taxas aplicadas anteriormente à avaliação reológica [5]. Uma forma de avaliar o comportamento tixotrópico é através de um gráfico de $\tau$ versus $\gamma^{\prime}$ (curva de fluxo), que é construído com um aumento da taxa de cisalhamento até um determinado valor, em seguida diminuindo até o ponto inicial. Nota-se que as curvas de subida (up curve) e de descida (down curve) não coincidem. Esta histerese é causada pela diminuição da viscosidade do fluido com o aumento do tempo de cisalhamento. Este efeito pode ou não ser reversível. Paralelamente pode ser observada a mesma histerese na curva de viscosidade, $\eta$ versus $\gamma^{\prime}[3,6]$.

As características reológicas das argilas comumente utilizadas em cerâmica dependem do tipo e da quantidade dos argilominerais presentes, assim como dos minerais acessórios.

Os argilominerais mais comuns presentes nas argilas usadas no setor cerâmico são aqueles dos grupos da caulinita, illita, esmectita, vermiculita e clorita. A caulinita não permite a presença de água intercamadas devido a sua estrutura mineralógica [7]. Este fato mostra que a caulinita não manifesta um comportamento reológico do tipo plástico, portanto não se observa uma tensão de escoamento, em condição reológica desfavorável [6]. Os argumentos relativos às illitas são limitados devido ao baixo grau de pureza quando comparado com o da caulinita. Ao se considerar uma argila illítica-caulinítica, supondo a ausência de outros minerais e impurezas, há um aumento do comportamento plástico do sistema em relação ao da caulinita, com tixotropia mais acentuada e aumento da viscosidade aparente. Da mesma forma, considerando uma argila illítica-montmorillonítica e confrontando-a com uma montmorillonítica, nota-se que os valores de viscosidade aparente são menores, assim como a tensão de escoamento e a tixotropia do sistema [6]. A montmorillonita apresenta um comportamento reológico característico, que é muito bom e requerido em várias aplicações, possui elevada tixotropia e tensão de escoamento definida, ou seja, uma tensão mínima para iniciar o escoamento, o que é inadequado para o processamento de dispersões com fins cerâmicos, como massas preparadas por via úmida [6]. Em comparação com os minerais descritos acima, torna-se muito difícil definir as características reológicas das cloritas. Isto se deve ao fato de que sendo a clorita um dos argilominerais mais comuns, desde sua gênese, sempre se encontra acompanhada das illitas ou esmectitas [6].

A Formação Corumbataí é a unidade geológica onde é encontrada a matéria-prima utilizada pelas indústrias cerâmicas do pólo de Santa Gertrudes. Nesta formação, o argilomineral predominante é a illita, que pode estar associada à clorita e suas variações estruturais na porção basal ou à montmorillonita na parte superior [8]. Apesar de sua importância tecnológica, o comportamento reológico dessa formação ainda não foi estudado em detalhe, assim este trabalho tem por objetivo estudar barbotinas preparadas com argilas da Formação Corumbataí, selecionadas de acordo com as variações observadas na região.

\section{MATERIAIS E MÉTODOS}

Foram selecionadas três áreas para estudo: mina Cruzeiro, jazida Morro Alto e mina Partezani. Localizadas na região do Pólo de Santa Gertrudes, interior de S. Paulo e distantes uma das outras, de forma a tornar ampla a área de estudo tanto horizontal como verticalmente da Formação Corumbataí.

As amostras da mina Cruzeiro receberam a seguinte denominação: Cruz B2, Cruz B3, Cruz B4, Cruz B5 e Cruz $\mathrm{B} 6$, sendo que a primeira corresponde à bancada da base da mina e a última ao topo. As amostras da jazida Morro Alto receberam a denominação Ara B1 (base) e Ara B2 (topo) e as da mina Partezani, Part B1 (base) e Part B2 (topo). A coleta das amostras foi feita com perfuratriz de acionamento pneumático, o material foi cuidadosamente recolhido, armazenado em saco plástico e identificado. As amostras para barbotina foram preparadas inicialmente através da seguinte seqüência: secagem em estufa (temperatura até $110^{\circ} \mathrm{C}$ ), quarteamento (em quarteador tipo Jones), moagem a seco em moinho de martelos e peneiramento em malha ASTM N³5 (abertura 0,5 mm). Em seguida, foi medida a umidade deste material, que foi moído à úmido em moinho de bolas planetário (300 g por amostra) para se obter uma 
barbotina com densidade de $1,65 \mathrm{~g} / \mathrm{mL}$ e teor de sólido de $63 \%$. O defloculante utilizado foi o silicato de sódio $\mathrm{Na}_{2} \mathrm{O}$ $\left(\mathrm{SiO}_{2}\right)$, densidade de $1,57 \mathrm{~g} / \mathrm{cm}^{3}$.

Não houve uma nova secagem a $\left(110^{\circ} \mathrm{C}\right)$ conforme mencionado anteriormente, apenas antes da moagem a seco para reduzir a umidade para $4 \%$ para facilitar a moagem a seco, tal procedimento também não é realizado em escala industrial, e pode ocorrer diferença, principalmente pelo fato destas argilas serem plásticas. Como pode ser visto nos resultados as argilas acabaram mostrando grande diferença, que foi o objetivo.

A granulometria das amostras foi controlada através do tempo de moagem e foi verificada pelo resíduo medido em peneira malha ASTM 200 (abertura 0,074 mm), buscando uma quantidade de material retido próximo a $4 \%$. Foi obtida a curva de distribuição granulométrica por difração a laser, no equipamento Mastersizer 2000 Malvern, com 30 s de ultra-som para dispersão e bomba a $2100 \mathrm{rpm}$. Para a determinação das curvas de fluxo e de viscosidade foi necessário fazer as medições dos seguintes parâmetros reológicos: viscosidade, tensão de cisalhamento e taxa de cisalhamento, através da variação contínua da taxa de cisalhamento (velocidade de rotação do spindle), inicialmente crescente e depois decrescente, com intervalo de $10 \mathrm{~s}$, foi feita uma agitação prévia da barbotina. Foi utilizado um viscosímetro rotacional Brookfield modelo LV-DV II+ programável, com o dispositivo de pequenas amostras SC418/13, que permitiu medidas numa faixa adequada para estas barbotinas, e o software Wingather 32 para modelamento matemático.

Para a caracterização mineralógica foi utilizado um difratômetro de raios X Siemens D5000, medidas com radiação $\operatorname{Co}(\lambda=1,7893 \AA), 2^{\circ}(2 \theta) /$ min, e foram comparados os difratogramas da amostra total com amostras da fração fina $(<2 \mu \mathrm{m})$ seca ao ar, glicolada e queimada.

\section{RESULTADOS E DISCUSSÃO}

Dentre as formas de análises que podem ser feitas com os dados coletados, optou-se por obter a curva de tensão de cisalhamento $\mathrm{x}$ taxa de cisalhamento (curva de fluxo) e a curva de viscosidade $\mathrm{x}$ taxa de cisalhamento (curva de viscosidade), por meio das quais se torna possível fazer uma análise comparativa entre as amostras dos comportamentos dependentes do tempo, assim como do comportamento independente do tempo.

Na Fig. 1 são apresentadas as curvas de fluxo das amostras de argilas das bancadas Cruz B2, Cruz B3, Cruz B4, Cruz B5 e Part B1, que se apresentaram menos alteradas por intemperismo. Verifica-se um comportamento levemente pseudoplástico, através da curvatura, além de uma histerese entre a curva de subida e a de descida da taxa de cisalhamento, mostrando as diferenças de comportamentos tixotrópicos. Esta mesma análise é válida para as amostras das bancadas Cruz B6, Ara B1, Ara B2 e Part B2, que se mostraram mais alteradas pelo intemperismo (Fig. 2). A amostra Cruz B2 apresentou o melhor comportamento, com

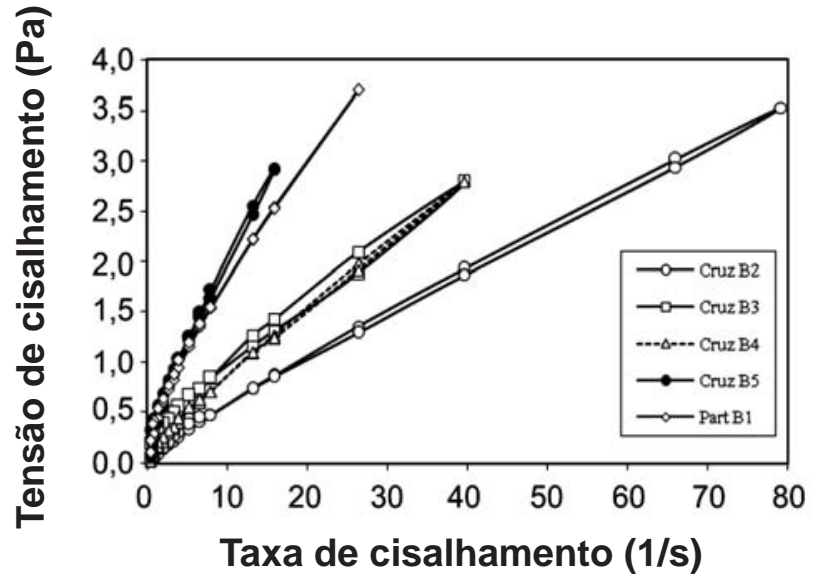

Figura 1: Curvas de fluxo das amostras que apresentaram menor alteração ao intemperismo.

[Figure 1: Flow curves of the samples which showed less weathering.]

baixa inclinação e pouca histerese, em seguida, as amostras Cruz B3 e Cruz B4 poderiam ser classificadas com um comportamento intermediário, diferenciando-se apenas na histerese que ocorre entre as curvas (tixotropia), e por fim as amostras Cruz B5 e Part B1 apresentaram os piores resultados com grande inclinação da curva.

Nota-se uma piora do comportamento reológico de todas as amostras da Fig. 2 em relação às amostras da Fig. 1, através do aumento das inclinações das curvas de fluxo e da histerese que é muito maior, de modo que a curva de descida retorna com valores maiores que a curva de subida, mostrando que estas barbotinas estão sofrendo o processo de gelificação com as taxas de cisalhamento que estão sendo aplicadas. Entre as amostras da Fig. 2, verifica-se que a Part B2 não possui um comportamento tão marcante quanto as demais, fato que está relacionado com o tempo de gelificação.

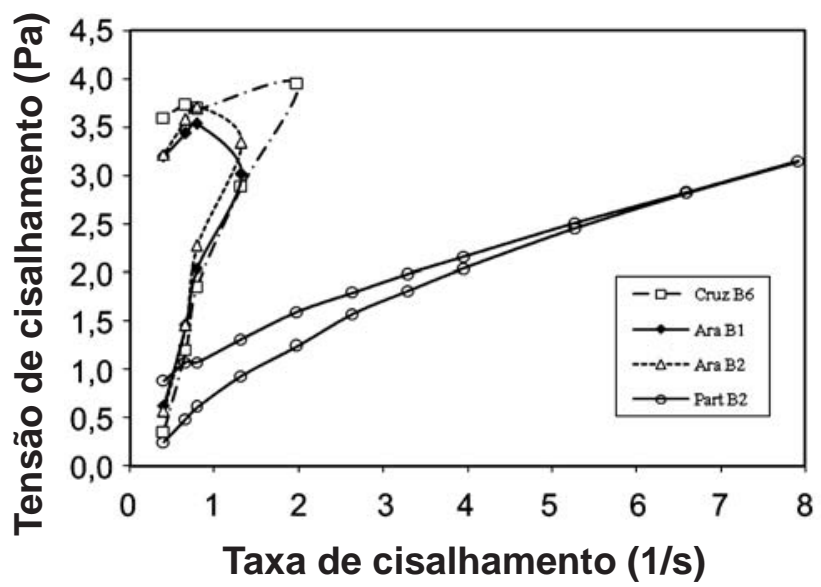

Figura 2: Curvas de fluxo das amostras que apresentaram maior alteração ao intemperismo.

[Figure 2: Flow curves of the samples which showed more weathering.] 
Tabela I - Comparação de parâmetros reológicos obtidos nas curvas de viscosidade.

[Table I - Comparison of rheological parameters obtained from viscosity curves.]

\begin{tabular}{lccccccccc}
\hline & $\begin{array}{l}\text { Cruz } \\
\text { B2 }\end{array}$ & $\begin{array}{l}\text { Cruz } \\
\text { B3 }\end{array}$ & $\begin{array}{l}\text { Cruz } \\
\text { B4 }\end{array}$ & $\begin{array}{l}\text { Cruz } \\
\text { B5 }\end{array}$ & $\begin{array}{l}\text { Cruz } \\
\text { B6 }\end{array}$ & $\begin{array}{l}\text { Ara } \\
\text { B1 }\end{array}$ & $\begin{array}{l}\text { Ara } \\
\text { B2 }\end{array}$ & $\begin{array}{l}\text { Part } \\
\text { B1 }\end{array}$ & $\begin{array}{l}\text { Part } \\
\text { B2 }\end{array}$ \\
\hline $\begin{array}{l}\text { Maior velocidade } \\
\text { (rpm) }\end{array}$ & 60 & 30 & 30 & 12 & 1,5 & 1 & 1 & 20 & 6 \\
$\begin{array}{l}\text { Viscosidade na maior velocidade } \\
\text { (mPa.s) }\end{array}$ & 44,5 & 70,4 & 70,5 & 183 & 1998 & 2283 & 2531 & 140,1 & 397,4 \\
$\begin{array}{l}\text { Viscosidade inicial } \\
\text { (mPa.s) }\end{array}$ & 70 & 120 & 60 & 239,9 & 889,8 & 1570 & 1430 & 279,9 & 619,9 \\
$\begin{array}{l}\text { Maior viscosidade } \\
\text { (mPa.s) }\end{array}$ & 220 & 519,9 & 249,9 & 799,8 & 9078 & 8078 & 8118 & 559,9 & 2230 \\
\hline
\end{tabular}

Tabela II - Análise das curvas de fluxo com modelo da lei das potências da equação (B).

[Table II - Analysis of the flow curves using the power law model from equation (B).]

\begin{tabular}{|c|c|c|c|c|c|c|c|c|c|}
\hline & $\begin{array}{l}\text { Cruz } \\
\text { B2 }\end{array}$ & $\begin{array}{l}\text { Cruz } \\
\text { B3 }\end{array}$ & $\begin{array}{l}\text { Cruz } \\
\text { B4 }\end{array}$ & $\begin{array}{l}\text { Cruz } \\
\text { B5 }\end{array}$ & $\begin{array}{l}\text { Cruz } \\
\text { B6 }\end{array}$ & Ara B1 & Ara B2 & Part B1 & Part B2 \\
\hline $\begin{array}{l}\text { Indice de Consistência } \\
\text { (mPa.s) }\end{array}$ & 69,8 & 118,7 & 95,4 & 283,2 & 1853 & 2359 & 2570 & 318,3 & 660,5 \\
\hline Índice de Fluxo & 0,91 & 0,91 & 0,96 & 0,89 & 1,45 & 1,32 & 1,50 & 0,77 & 0,81 \\
\hline Ajuste de Confiança (\%) & 94,9 & 91,1 & 86,5 & 90,7 & 77,6 & 88,5 & 86,1 & 92,0 & 91,4 \\
\hline
\end{tabular}

Tabela III - Comparação de valores de resíduos obtidos na moagem via úmida.

[Table III - Comparison of the residual particle size values obtained from the wet grinding.]

\begin{tabular}{llllllllll}
\hline & Cruz & Cruz & Cruz & Cruz & Cruz & Ara & Ara & Part & Part \\
B2 & B4 & B6 & B1 & B2 & B1 & B2 \\
\hline $\begin{array}{l}\text { Resíduo retido em } \\
\text { \#200 (\%) }\end{array}$ & 1,74 & 5,29 & 3,1 & 1,32 & 5,07 & 2,42 & 2,2 & 3,73 & 4,03 \\
$\begin{array}{l}\text { Fração superior a } \\
\text { \#200 (\%) método a } \\
\text { laser }\end{array}$ & 2,03 & 7,11 & 2,91 & 3,65 & 3,94 & 2,51 & 2,62 & 4,63 & 6,62 \\
\hline
\end{tabular}

Através das curvas de viscosidade foram escolhidos alguns parâmetros para a construção da Tabela I, com objetivo de facilitar a comparação dos resultados medidos de todas as amostras analisadas. Na Tabela II encontramse os resultados obtidos através da modelagem matemática utilizando-se a lei das potências da equação (B).

Comparando-se os valores das amostras da mina Cruzeiro na Tabela I verifica-se um aumento no parâmetro viscosidade na maior velocidade, assim como, no parâmetro maior viscosidade, da amostra Cruz B2 (base da mina) para a amostra Cruz B6 (topo da mina), sendo que as amostras desta mina apresentaram os menores valores de viscosidade medidos. As amostras Ara B1 e Ara B2, obtiveram valores muito próximos e muito altos, por último, a amostra Part
B1 (base) apresenta valores de viscosidade menores que a Part B2, porém estes valores podem ser classificados como intermediários, entre os valores obtidos para as amostras Cruz e Ara.

Verifica-se na Tabela II, que os resultados do parâmetro consistency index (k) na equação (B), justificam a análise feita para os dados da Tabela I. Nota-se que o flow index (n) da equação (B) é menor que 1 para quase todas as amostras, exceto para Cruz B6, Ara B1 e Ara B2, que apresentaram uma forte gelificação durante o ensaio.

Nota-se que os valores obtidos através das medidas de resíduo em peneira malha ASTM 200 para as diversas amostras estão em acordo com os valores obtidos nas curvas de distribuição granulométrica por difração a laser, e tais 
Tabela IV - Composição mineralógica da mina Cruzeiro, da jazida Morro Alto e mina Partezani. [Table IV - Mineralogical composition of Cruzeiro quarry, Morro Alto clay deposit and Partezani quarry.]

\begin{tabular}{llllllllll}
\hline & Cruz & Cruz & Cruz & Cruz & Cruz & Ara & Ara & Part & Part \\
& B2 & B3 & B4 & B5 & B6 & B1 & B2 & B1 & B2 \\
\hline Quartzo & $\square$ & $\square$ & $\square$ & $\square$ & $\square$ & $\square$ & $\square$ & $\square$ & $\square$ \\
Illita & $\square$ & $\square$ & $\square$ & $\square$ & $\square$ & $\square$ & $\square$ & $\square$ & $\square$ \\
Albita & $\square$ & $\square$ & $\square$ & $\square$ & $\square$ & $\square$ & $\square$ & $\square$ & $\square$ \\
Hematita & $\square$ & $\square$ & $\square$ & $\square$ & $\square$ & $\square$ & $\square$ & & $\square$ \\
Dolomita & & $\square$ & $\square$ & $\square$ & $\square$ & $\square$ & $\square$ & & \\
Clorita & $\square$ & $\square$ & $\square$ & & & & & $\square$ & $\square$ \\
Montmorillonita & & & & $\square$ & $\square$ & & $\square$ & & \\
Calcita & & & $\square$ & & $\square$ & $\square$ & $\square$ & $\square$ & $\square$ \\
Microclínio & & & & & & $\square$ & $\square$ & & \\
Analcima & & & & & & $\square$ & $\square$ & & \\
Interestratificados & & & $\square$ & $\square$ & $\square$ & $\square$ & $\square$ & $\square$ & $\square$ \\
\hline
\end{tabular}

resultados se aproximam ao valor de $4 \%$, proposto para o controle da moagem (Tabela III).

Na Tabela IV encontra-se a composição mineralógica das amostras estudadas, obtidas por difração de raios X. Em termos qualitativos, as argilas estudadas possuem uma mineralogia comum, constituída pelo argilomineral illita e os minerais quartzo e albita, ocorrendo o mineral calcita nas camadas do topo. O comportamento reológico menos favorável das amostras do topo da mina Cruzeiro, Cruz B5 e Cruz B6 pode estar relacionado à presença do argilomineral montmorillonita, assim como de interestratificados irregulares expansíveis como resultado de transições cloritamontmorillonita, que surgem ao mesmo tempo que desaparece a clorita [6]. As amostras da jazida Morro Alto, apresentaram uma mineralogia um pouco diferente das demais, pela presença da analcima e do microclínio e ausência da clorita, ocorrendo a montmorillonita apenas na amostra Ara B2, além da presença de interestratificados illita-montmorillonita, estes argilominerais são produtos intermediários de reações envolvendo membros iniciais e finais em estado de alteração, provocando o aumento da viscosidade nas duas amostras desta jazida $[9,10]$. As amostras da mina Partezani apresentam uma mineralogia muito semelhante às amostras superiores da mina Cruzeiro, embora não apresente a montmorillonita, a camada Part B2 mostrou grande tendência à gelificação, pela alteração das illitas e cloritas para os interestratificados com montmorillonita. A clorita é freqüentemente encontrada nas camadas menos alteradas, principalmente nas mais inferiores.

\section{CONCLUSÕES}

A determinação das curvas de fluxo e das curvas de viscosidade mostrou ser um método rápido e eficiente para se entender o comportamento reológico das barbotinas evidenciando a natureza físico-química das argilas.

Através das propriedades reológicas medidas se verifica uma piora no sentido da base para o topo (aumento de viscosidade, aumento do comportamento tixotrópico, tendência à rápida gelificação).

As amostras Cruz B2, Cruz B3, Cruz B4, Cruz B5 e Part B1 podem ser facilmente defloculadas com boa viscosidade ( $<250 \mathrm{mPa}$.s, na maior velocidade ou taxa de cisalhamento aplicada) para densidade de $1,65 \mathrm{~g} / \mathrm{mL}$, pois apresenta características de fluidez adequadas para a preparação de massa via úmida e nas quais predominam os argilominerais illita e clorita. As amostras Cruz B6, Ara B1, Ara B2 e Part B2 apresentaram uma forte tendência à gelificação e obtiveram valores muito altos de viscosidade (> $500 \mathrm{mPa}$.s, na maior velocidade ou taxa de cisalhamento aplicada) para a mesma densidade de $1,65 \mathrm{~g} / \mathrm{mL}$, podendo fazer parte de uma formulação de massa via úmida, apenas em quantidades muito reduzidas.

Em termos de mineralogia há uma predominância da illita , quartzo e albita, nas camadas do topo é freqüente o aparecimento da calcita e da montmorillonita e nas camadas da base o aparecimento da clorita, porém cada mina tem sua própria característica.

De maneira geral, todas as amostras ensaiadas podem ser utilizadas na preparação de massa via úmida, desde que a contribuição delas seja devidamente controlada.

\section{REFERÊNCIAS}

[1] I. R. de Oliveira, A. R. Studart, R. G. Pileggi, V. C. Pandolfelli, Dispersão e empacotamento de partículas - princípios e aplicações em processamento cerâmico, Fazendo Arte Editorial, S. Paulo (2000) 11.

[2] Brookfield Eng. Labs, More solutions to sticky problems. Disponível em www.brookfieldengineering.com.htm, (2006) 14. 
[3] G. Scharamm, Reologia e reometria: fundamentos teóricos e práticos, S. Paulo, Artliber Editora (2006) 27.

[4] P. S. Santos, Ciência e tecnologia de argilas, $2^{\mathrm{a}}$. Ed., Vol. 1 e 2, Edgard Blücher Ltda. e EDUSP, S. Paulo (1989) 109.

[5] H. van Olphen, An introduction to clay colloid chemistry, For Clay Technologists, Geologists and Soil Scientists. John Wiley \& Sons, EUA (1963) 132.

[6] A. Ravaglioli, Reologia Ceramica Applicata, Società Ceramica Italiana, Faenza Ed., Bologna 51 (1989) 133.

[7] W. A. Deer, R. A. Howie, J. Zussman, Minerais constituintes das rochas: uma introdução, Lisboa, Fundação Calouste Gulbenkian (1966) 372.

[8] A. Zanardo, M. M. T. Moreno, C. D. Roveri, A. C. A. Prado, M. R. Masson, J. F. M. Motta, L. Ibrahim. IX World Cong. Ceram. Tile Quality - Qualicer, Castellón, Espanha 3 (2006) 181.

[9] J. Środoń, Ann. Rev. Earth Planet. Sci. 27 (1999) 19.

[10] A. C. A. Prado, C. D. Roveri, R. R. Rocha, A. Zanardo, M. M. T. Moreno, J. F. M. Motta, A. P. M. Menegazzo, REM - Revista Escola de Minas 60, 4 (2007) 613.

(Rec. 13/09/2007, Rev. 07/02/2008, Ac. 11/04/2008) 